\title{
Use of probiotic Lactobacillus preparation to prevent diarrhoea associated with antibiotics: randomised double blind placebo controlled trial
}

\author{
Mary Hickson, research dietitian, ${ }^{1}$ Aloysius L D'Souza, research fellow, ${ }^{2}$ Nirmala Muthu, research nurse, ${ }^{3}$ \\ Thomas R Rogers, professor of clinical microbiology and honorary consultant (Hammersmith Hospitals NHS \\ Trust), ${ }^{4}$ Susan Want, clinical scientist, ${ }^{5}$ Chakravarthi Rajkumar, senior lecturer, ${ }^{2}$ Christopher J Bulpitt, \\ professor of geriatric medicine ${ }^{2}$
}

${ }^{1}$ Nutrition and Dietetic Research Group, Faculty of Medicine, Imperial College, London W12 OHS

${ }^{2}$ Medicine for the Elderly, Faculty of Medicine, Imperial College.

London

${ }^{3}$ Hillingdon Hospital, Uxbridge

${ }^{4}$ Department of Infectious

Diseases and Immunity, Faculty of

Medicine, Imperial College School

of Medicine, London

${ }^{5}$ Microbiology Department,

Hammersmith Hospital NHS Trust, London

Correspondence to: M Hickson, Department of Nutrition and

Dietetics, Charing Cross Hospital,

London W6 8RF

mhickson@hhnt.nhs.uk

doi:10.1136/bmj.39231.599815.55

\section{ABSTRACT}

Objective To determine the efficacy of a probiotic drink containing Lactobacillus for the prevention of any diarrhoea associated with antibiotic use and that caused by Clostridium difficile.

Design Randomised double blind placebo controlled study.

Participants 135 hospital patients (mean age 74 ) taking antibiotics. Exclusions included diarrhoea on admission, bowel pathology that could result in diarrhoea, antibiotic use in the previous four weeks, severe illness, immunosuppression, bowel surgery, artificial heart valves, and history of rheumatic heart disease or infective endocarditis.

Intervention Consumption of a $100 \mathrm{~g}(97 \mathrm{ml})$ drink containing Lactobacillus casei, L bulgaricus, and Streptococcus thermophilus twice a day during a course of antibiotics and for one week after the course finished. The placebo group received a longlife sterile milkshake.

Main outcome measures Primary outcome: occurrence of antibiotic associated diarrhoea. Secondary outcome: presence of $C$ difficile toxin and diarrhoea.

Results $7 / 57$ (12\%) of the probiotic group developed diarrhoea associated with antibiotic use compared with $19 / 56(34 \%)$ in the placebo group ( $P=0.007)$. Logistic regression to control for other factors gave an odds ratio 0.25 ( $95 \%$ confidence interval 0.07 to 0.85 ) for use of the probiotic, with low albumin and sodium also increasing the risk of diarrhoea. The absolute risk reduction was $21.6 \%$ (6.6\% to $36.6 \%$ ), and the number needed to treat was 5 ( 3 to 15 ). No one in the probiotic group and $9 / 53$ (17\%) in the placebo group had diarrhoea caused by $C$ difficile $(P=0.001)$. The absolute risk reduction was $17 \%(7 \%$ to $27 \%$ ), and the number needed to treat was 6 (4 to 14 ). Conclusion Consumption of a probiotic drink containing $L$ casei, $L$ bulgaricus, and $S$ thermophilus can reduce the incidence of antibiotic associated diarrhoea and C difficile associated diarrhoea. This has the potential to decrease morbidity, healthcare costs, and mortality if used routinely in patients aged over 50 .

Trial registration National Research Register N0016106821.

\section{INTRODUCTION}

There is increasing evidence that probiotics are beneficial in a range of gastrointestinal conditions, including infectious diarrhoea and that related to antibiotic use. ${ }^{1}$ Probiotics are defined as "live micro-organisms which when administered in adequate amounts confer a health benefit on the host"2 and include Streptococcus thermophilus, Enterococcus species, Saccharomyces species, and various species of lactobacilli and bifidobacteria.

Diarrhoea associated with antibiotic use and caused by Clostridium difficile is a complication of treatment with antimicrobial agents and occurs in about $5-25 \%$ of patients. ${ }^{3}$ C difficile is responsible for around $15-25 \%$ of all cases of diarrhoea associated with antibiotic use, most occurring in older patients, usually in the two to three weeks after cessation of antibiotic treatment. ${ }^{4}$ Lactobacilli, bifidobacteria, and Streptococcus species have all been evaluated for the prevention or treatment of diarrhoea associated with antibiotic use and found to be safe. ${ }^{5}$ Several reviews support benefit but still call for large placebo controlled trials to determine species and dose effectiveness for prevention, to show effectiveness in preventing diarrhoea caused by $C$ difficile, and to establish effect on length of hospital stay and cost effectiveness. ${ }^{6-8}$

We undertook a randomised double blind, placebo controlled trial of a commercially available probiotic preparation in older patients in hospital who were receiving antibiotics.

\section{METHODS}

The hypothesis was that a probiotic Lactobacillus preparation would reduce the incidence of antibiotic associated diarrhoea and $C$ difficile associated diarrhoea. Our primary outcome was the occurrence of diarrhoea, which was recorded by the nursing staff and authenticated by the researchers. Diarrhoea was defined as more than two liquid stools a day for three or more days in quantities in excess of normal for each patient. The secondary outcome was the occurrence of $C$ difficile infection, defined as an episode of diarrhoea combined with the detection of toxins A or B, or both, 
from a stool sample (enzyme immunoassay kit, Meridian Bioscience, OH, USA).

\section{Participants}

We recruited patients from three London hospitals: Hammersmith, Charing Cross, and Hillingdon. Patients were recruited mainly from orthopaedic, medical, and care of the elderly wards, and included inpatients aged over 50 who were prescribed antibiotics (single or multiple antibiotics, oral or intravenous) and were able to take food and drink orally. Initially patients had to be able to give written informed consent, though half way through the trial we received ethical approval to recruit patients with cognitive impairment. Nevertheless, only seven patients had an abbreviated mental test score ${ }^{9}$ less than 7 (four in the control group and three in the probiotic group).

Our exclusion criteria were diarrhoea on admission or within the preceding week, reported recurrent diarrhoea, or bowel pathology that could result in diarrhoea; intake of high risk antibiotics (clindamycin, cephalosporins, aminopenicillins) or more than two courses of other antibiotics in the past four weeks to exclude pre-existing diarrhoea associated with antibiotic use; severe life threatening illness, immunosuppression, bowel surgery, artificial heart valve, history of rheumatic heart disease, or history of infective endocarditis ${ }^{5}$; regular probiotic treatment before admission; and lactose intolerance or intolerance to dairy products.

\section{Interventions}

The treatment group received a probiotic yoghurt drink (Actimel, Danone, France) containing Lactobacillus case $\mathrm{DN}-114001$ (L casei imunitass) $\left(1.0 \times 10^{8}\right.$ colony

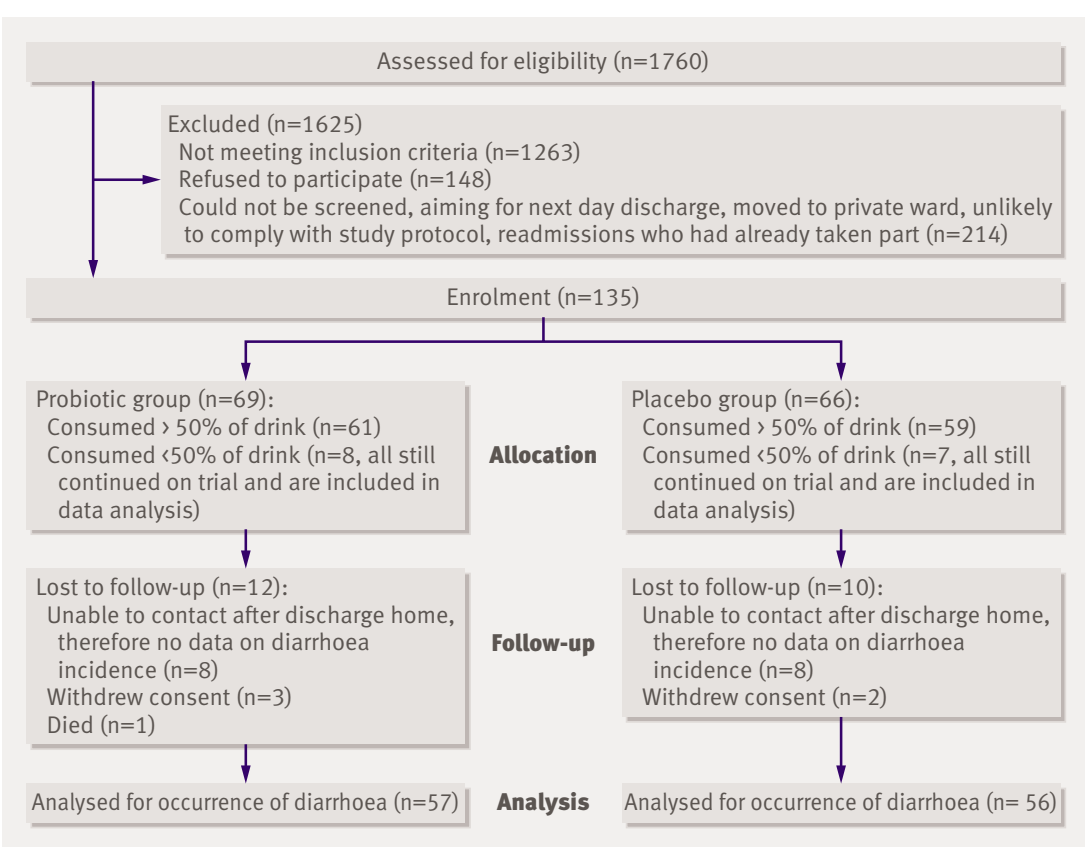

Flow chart showing numbers of patients at each stage of the trial forming units $/ \mathrm{ml})$, S thermophilus $\left(1.0 \times 10^{8} \mathrm{cfu} / \mathrm{ml}\right)$, and $L$ bulgaricus $\left(1.0 \times 10^{7} \mathrm{cfu} / \mathrm{ml}\right)$. The placebo group received a longlife, sterile milkshake (Yazoo, Campina, Netherlands). We carried out general lactobacillus counts on a sample of the probiotic drinks to ensure they were active. L casei imunitass has been shown to travel through the human gut and survive into the large intestine, ${ }^{10}$ thus meeting one criterion of a probiotic. Participants began using the drinks within 48 hours of starting antibiotic therapy and continued doing so for one week after they stopped taking antibiotics. They drank $100 \mathrm{~g}(97 \mathrm{ml})$ twice daily half an hour before or one to two hours after meals. Researchers verified participants' consumption and recorded missed or refused drinks to assess compliance.

\section{Study plan}

The admitting medical team identified potential patients who had been prescribed antibiotics and the researchers approached them within 48 hours of the first antibiotic dose. Once they obtained informed consent, they collected baseline data and prescribed the randomised study drink. The hospital pharmacy dispensed the drink. A baseline stool sample was collected to screen for asymptomatic $C$ difficile carriage. Bowel movements were monitored with stool charts, which were checked every weekday for accuracy. When there was evidence of diarrhoea a stool sample was analysed for $C$ difficile toxin.

Once the antibiotic course was finished a final week of study drink was dispensed and a final follow-up date fixed for four weeks later. Patients who were discharged taking antibiotics were provided with enough drink on discharge to cover the period they had to take antibiotics plus one week. Researchers followed up participants for four weeks from discharge with weekly phone calls to ask about diarrhoea and compliance. If participants had diarrhoea, the researchers collected a further stool sample to check for $C$ difficile toxin.

\section{Sample size}

With $\alpha=0.05$ and a power of $90 \%$ to detect an absolute difference of $20 \%$ between the proportion of patients with antibiotic associated diarrhoea in the placebo (assumed at 30\%) and probiotic (assumed at 10\%) groups we estimated that we needed a sample size of 164 (82 in each group).

\section{Randomisation}

An independent statistician generated the random allocation sequence, which was stratified for hospital, sex, and two age groups (50-69 and $\geq 70)$. The sequence was given to the pharmacy on each site.

\section{Blinding}

Actimel is sold in $100 \mathrm{~g}$ white plastic bottles with removable labels; Yazoo is packaged similarly but in $200 \mathrm{ml}$ bottles. We chose Yazoo as placebo because it looks identical in colour and consistency to Actimel but is an ultra high temperature treated product and has no 
bacterial content. The pharmacies removed the commercial labels, then applied study labels to identify the patient, the drink's "use by" date, and storage instructions. We could not find a placebo in an identical bottle to Actimel.

Patients and researchers were blind to the study drink as they did not see the bottle the drink came in. Nursing staff dispensed the drinks and were instructed to pour $100 \mathrm{ml}$ into a cup for the patient; they were not told which bottle contained which drink. Older people in the UK are not generally familiar with these products, but it is possible some patients might have recognised the taste. However, we had excluded people who regularly took this or other probiotic products from the study.

Potential bias through unblinding was possible but unlikely, and the outcome measure was checked and agreed between two or more people. Microbiology staff who were blind to the study grouping assessed

\begin{tabular}{|c|c|c|}
\hline & Probiotic $(n=69)$ & Control $(n=66)$ \\
\hline Women & $39(57)$ & $34(52)$ \\
\hline White European & $61(88)$ & $59(89)$ \\
\hline Mean (SD) age (years) & $73.7(11.1)$ & $73.9(10.5)$ \\
\hline Median (IQR) BMI & $25.5(20.6-31.5)$ & $23.7(19.7-30.5)$ \\
\hline Mean (SD) albumin $(\mathrm{g} / \mathrm{l})$ & $33.2(5.3)$ & $32.7(6.1)$ \\
\hline Median (IQR) white cell count (/I) & $9.0(6.8-13.3)$ & $9.5(7.0-13.1)$ \\
\hline Median (IQR) plasma sodium (mmol/l) & $138.0(135-140)$ & $137.0(135-139)$ \\
\hline Median (IQR) C reactive protein $(\mathrm{mg} / \mathrm{l})$ & $37.0(13.0-118.0)$ & $46.0(15.8-140.0)$ \\
\hline Median (IQR) thyroid stimulating hormone $(\mathrm{mU} / \mathrm{l})$ & $1.17(0.56-2.09)$ & $0.9(0.6-1.74)$ \\
\hline Median (IQR) thyroxine (pmol/l) & $15.5(14.0-17.2)$ & $15.3(13.0-16.7)$ \\
\hline \multicolumn{3}{|l|}{ Median (IQR) length of stay (days): } \\
\hline Before randomisation & $1.0(1.0-2.0)$ & $1.0(1.0-2.0)$ \\
\hline After randomisation & $9.0(5.0-16.0)$ & $8.0(4.5-14.0)$ \\
\hline Alcohol drinkers & $34(49)$ & $33(50)$ \\
\hline Median (IQR) alcohol units/week in drinkers & $6(2-12)$ & $9(3-21)$ \\
\hline Smokers & $19(28)$ & $11(17)$ \\
\hline Mean (SD) No of cigarettes/week in smokers & $82(66)$ & $102(87)$ \\
\hline \multicolumn{3}{|l|}{ No of antibiotics prescribed: } \\
\hline 1 & $40(58)$ & $34(52)$ \\
\hline 2 & $26(38)$ & $29(44)$ \\
\hline 3 & $3(4)$ & $3(5)$ \\
\hline \multicolumn{3}{|l|}{ Risk of antibiotic causing diarrhoea*: } \\
\hline Low & $0(0)$ & $1(1.5)$ \\
\hline Medium & $26(38)$ & $19(29)$ \\
\hline High & $43(62)$ & $46(70)$ \\
\hline \multicolumn{3}{|l|}{ Indication for antibiotics: } \\
\hline Respiratory tract infection & $33(48)$ & $33(50)$ \\
\hline Prophylaxis before/after surgery & $17(25)$ & $17(26)$ \\
\hline Urinary tract infection & $10(15)$ & $10(15)$ \\
\hline Other† & $7(10)$ & $5(8)$ \\
\hline Missing & $2(3)$ & $1(2)$ \\
\hline
\end{tabular}

$\mathrm{IQR}=$ interquartile range; $\mathrm{BMI}=$ body mass index.

*Low risk=metronidazole and parenteral aminoglycosides (gentamicin); medium risk=tetracyclines

(oxytetracycline), sulphonamides (trimethoprim), macrolides (azithromycin, clarithromycin, erythromycin),

quinolones (ciprofloxacin); high risk=aminopenicillin (amoxicillin, benzylpenicillin, co-amoxiclav, flucloxacillin), cephalosporins (cefalexin, ceftazidime, cefuroxime) ${ }^{11}$

†Cellulitis (5), ulcer (2), pressure sore (1), sepsis (1), sore throat (1), cholecystitis (2). occurrence of $C$ difficile by analysis of a stool sample from patients who had diarrhoea.

\section{Statistical methods}

We used Fisher's exact test to compare rates of antibiotic associated diarrhoea and $C$ difficile associated diarrhoea and logistic regression (block entry with removal of non-significant variables) to establish which factors influenced the occurrence of diarrhoea and to estimate the adjusted odds ratio for treatment effect.

\section{RESULTS}

From November 2002 to January 2005, 135 patients entered the study (figure). The most common reason for exclusion (61\% of patients) was the likelihood of diarrhoea from causes unrelated to antibiotics. A further $21 \%$ of patients were excluded because of possible safety concerns, and $18 \%$ were not able to give informed consent nor were relatives willing or available to give assent. Only one patient was excluded because of a dairy allergy.

There were no reported adverse events related to the study drinks. In the 24 samples we tested for lactobacillus the mean count was $2.2 \times 10^{8} \mathrm{cfu} / \mathrm{ml}$ (range $\left.0.35 \times 10^{8}-4.6 \times 10^{8}\right)$. We found no bacterial content in the Yazoo samples.

There were no clinically important differences between the two groups at baseline (table 1). Most patients received one antibiotic, but about $40 \%$ received two (table 1). The most common reasons for antibiotic use were respiratory infection $(49 \%)$ or prophylaxis before or after surgery (25\%) (usually orthopaedic). Compliance was assessed by the percentage of prescribed drinks that were consumed: 75\% (interquartile range 55-91\%) of the probiotic and 79\% (63$94 \%$ ) of the control drink. The main reason for compliance rates falling below 100\% were delivery and distribution failures, rather than palatability. One patient in each group was positive for $C$ difficile toxin at baseline but had no diarrhoea; neither patient subsequently developed diarrhoea.

The primary analysis was intention to treat and included all patients with available end point data (table 2). We could not complete follow-up on 16\% (22/135; 12 in probiotic group, 10 in placebo group) as we were unable to contact them at home despite numerous phone calls and written communications (16) or they had withdrawn (6) from the study, thus the analysis for occurrence of antibiotic associated diarrhoea included 113 patients (56 in control and 57 in probiotic group). Four patients were not tested for $\mathrm{C}$ difficile (one in probiotic group, three in control group) and thus were not included in the analysis for occurrence of diarrhoea associated with $C$ difficile. We found a significant reduction in both the incidence of antibiotic associated diarrhoea $(\mathrm{P}=0.007)$ and $C$ difficile associated diarrhoea $(\mathrm{P}=0.001)$ in the probiotic group. The absolute risk reduction for occurrence of antibiotic associated diarrhoea was 22\% (95\% confidence interval $7 \%$ to $37 \%$ ), and the number needed to 
Table 2 Total number of cases of antibiotic associated diarrhoea (including cases positive for $C$ difficile) and proportion who were positive or negative for $C$ difficile toxin (all patients followed up in hospital and after discharge)

\begin{tabular}{|c|c|c|c|}
\hline & Probiotic & Control & Pvalue* \\
\hline \multicolumn{4}{|l|}{ Diarrhoea } \\
\hline Yes & $7(12)$ & $19(34)$ & \multirow{2}{*}{0.007} \\
\hline No & $50(88)$ & $37(66)$ & \\
\hline No of patients & $57 \dagger$ & $56 \dagger$ & \\
\hline \multicolumn{4}{|l|}{ C difficile toxin } \\
\hline Positive & 0 & $9(17)$ & \multirow{2}{*}{0.001} \\
\hline Negative & $56(100)$ & $44(83)$ & \\
\hline No of patients & $56 \neq$ & $53 \ddagger$ & \\
\hline
\end{tabular}

*Fisher's exact test.

$\dagger 22 / 135$ patients lost to follow-up or withdrew.

$\ddagger 4 / 113$ patients not tested for $C$ difficile.

treat was 5 (3 to 15). For $C$ difficile associated diarrhoea the figures were $17 \%(7 \%$ to $27 \%)$ and 6 (4 to 14$)$.

Tables 3 shows the result of logistic regression of antibiotic associated diarrhoea as the dependent variable with treatment group, age, sex, indication for antibiotics, number of antibiotics, smoking, alcohol consumption, body mass index, serum albumin, thyroxine, white cell count, $\mathrm{C}$ reactive protein, and plasma concentrations of creatinine, potassium, and sodium as covariates. Every $1 \mathrm{~g} / \mathrm{l}$ increase in albumin concentration was associated with an $18 \%$ reduction in the odds of diarrhoea, and every $1 \mathrm{mmol} / \mathrm{l}$ increase in sodium concentration was associated with a $16 \%$ reduction. After adjustment for these variables the probiotic treatment reduced the odds of diarrhoea by $75 \%$. The small number of cases of $C$ difficile associated diarrhoea made logistic analysis inappropriate.

Additional information is available on bmj.com.

\section{DISCUSSION}

Twice daily intake of a probiotic drink containing $L$ casei, $L$ bulgaricus, and $S$ thermophilus for one week longer than the duration of antibiotic treatment can prevent diarrhoea associated with antibiotic use and that caused by $C$ difficile. There were no adverse events and the drink was well accepted. This is a major advance on previous meta-analyses, which called for further definitive trials and expressed considerable doubt as to the efficacy in preventing $C$ difficile associated diarrhoea. ${ }^{6-8}$

\section{Strengths and weaknesses}

This study was adequately powered for the large treatment effect in antibiotic associated diarrhoea and used a probiotic freely available in the UK. Additionally, we

Table 3| Risk model for prediction of antibiotic associated diarrhoea with logistic regression

Odds ratio $(95 \% \mathrm{Cl})$

Treatment (probiotic $=0$, placebo $=1$ ) 0.25 (0.07 to 0.85$)$

Plasma sodium 0.84 (0.75 to 0.95$)$

Serum albumin $0.82(0.73$ to 0.92$)$ were able to show the bacterial content of the product, included a four week absence of antibiotic use before enrolment, and tested for $C$ difficile toxin in all patients with diarrhoea.

In vitro studies have shown that different bacterial strains work differently, ${ }^{12}$ and previous trials have produced conflicting results ${ }^{13-19}$; thus other strains may not produce the same beneficial effect as the strains tested here. We could not establish which bacteria species are effective from the three strains in this probiotic drink; the three species may be working synergistically to prevent diarrhoea or alternatively one species may be more effective than another. Therefore, our results cannot be extrapolated to other probiotic products, which must be tested in similar trials. ${ }^{20}$

An obvious methodological weakness is the possibility of unblinding of the researchers and patients. We did, however, take precautions to avoid unblinding and, most importantly, several people confirmed the presence of diarrhoea. The loss to follow-up of $16 \%$ of the study population is also a weakness, though the numbers lost in each group were similar (12 in the probiotic group and 10 in the control group) and should not significantly alter the results.

The figure illustrates the difficulties in recruitment for this study; of 1760 patients screened only 135 took part. This raises questions about whether the results are generalisable. Some patients were excluded to prevent inclusion of diarrhoea from other causes and some patients were excluded because of a lack of informed consent; these factors are irrelevant in routine use and amounted to $79 \%$ of exclusions in this trial Others were excluded to ensure safety, and $21 \%$ were excluded on these grounds. Infections such as endocarditis or bacteraemia are rare, and recent safety reviews have questioned whether probiotics were actually the cause of infection in some documented cases. ${ }^{5}$ Nevertheless, we were cautious when recruiting for this study. Such strict criteria, however, may not be needed in practice. The high refusal rate (148) is unsurprising as we were approaching mainly elderly patients early in their hospital stay. The need for extensive data collection, monitoring, and collection of stool samples will certainly have discouraged some patients. Should probiotics become routinely used in hospitals patients are far more likely to accept it as an established rather than a trial treatment. Thus, we think the results can be generalised to any older patients in hospital, taking into account safety concerns for specific patients.

\section{Costs}

Using the numbers needed to treat (5 for antibiotic associated diarrhoea, 6 for $C$ difficile associated diarrhoea) we calculated the cost to prevent one case of antibiotic associated diarrhoea. The estimated average cost of the probiotic was $£ 10(€ 14.8 ; \$ 20)$ per patient (assuming an average antibiotic course of 10 days plus a further seven days of probiotics, and using current retail prices for Actimel, about $£ 0.30$ (€0.44; \$0.60) each). The cost to prevent one case would therefore be $£ 50(€ 74 ; \$ 100)$ for antibiotic associated diarrhoea 


\section{WHAT IS ALREADY KNOWN ON THIS TOPIC}

Probiotics may prevent diarrhoea associated with antibiotics and Clostridium difficile

Adequately powered, double blind randomised controlled trials are needed to assess the efficacy of specific probiotic strains

Evidence for the efficacy of probiotic products readily available in the UK is lacking

\section{WHAT THIS STUDY ADDS}

Consumption of a readily available probiotic drink containing Lactobacillus casei, $L$ bulgaricus, and Streptococcus thermophilus, twice a day during a course of antibiotics and for one week afterwards, reduces the incidence of diarrhoea associated with antibiotic use and $C$ difficile

Compliance with the probiotic drink was good

The cost to prevent one case of diarrhoea was $£ 50(€ 74 ; \$ 100)$ and $£ 60(€ 87.5 ; \$ 120)$ to prevent one case of $C$ difficile
1 Sullivan A, Nord CE. Probiotics and gastrointestinal diseases. J Intern Med 2005;257:78-92.

$2 \mathrm{FAO} / \mathrm{WHO}$ Expert Consultation Group. Health and nutritional properties of probiotics in food including powder milk with live lactic acid bacteria. Geneva: WHO, 2007.

3 Bergone-Berezin E. Treatment and prevention of antibioticassociated diarrhea. Int J Antimicrob Agents 2000;16:521-6.

4 Barbut F, Petit JC. Epidemiology of Clostridium difficile-associated infections. Clin Microbiol Infect 2001;7:405-10.

5 Boyle RJ, Robins-Browne RM, Tang ML. Probiotic use in clinical practice: what are the risks? Am J Clin Nutr 2006;83:1256-64.

6 Cremonini F, Di Caro S, Nista EC, Bartolozzi F, Capelli G, Gasbarrini G, et al. Meta-analysis: the effect of probiotic administration on antibiotic-associated diarrhoea. Aliment Pharmacol Ther 2002;16:1461-7.

7 McFarland LV. Meta-analysis of probiotics for the prevention of antibiotic associated diarrhea and the treatment of Clostridium difficile disease. Am J Gastroenterol 2006;101:812-22.

8 D'Souza AL, Rajkumar C, Cooke J, Bulpitt CJ. Probiotics in prevention of antibiotic associated diarrhoea: meta-analysis. BMJ 2002;324:1361.

9 Hodkinson HM. Evaluation of a mental test score for assessment of mental impairment in the elderly. Age Ageing 1972;1:233-8.

and $£ 60$ (€89; \$120) for C difficile associated diarrhoea, excluding dispensing and nursing costs. Evidence suggests that additional treatment costs per patient for $C$ difficile associated diarrhoea are on average $\$ 3669$ $(£ 1835 ; € 2738)$ in the United States ${ }^{21}$ and $£ 4000$ $(€ 5920 ; \$ 8000)$ in the United Kingdom ${ }^{22}$, mainly because of increased length of stay in hospital but also because of the use of vancomycin. Clearly substantial savings could be made by the routine use of probiotics.

We thank Winston Banya for statistical advice and conducting the randomisation; Sheila Bacon, Paula Brown, and Linda Wedlake for assistance with data collection; Edward Pawley, Lucy O'Driscoll, Regina Storch, Kuldip Dehal, and the pharmacy departments at each hospital for management and dispensing of the study drinks; the nursing staff on all the wards involved for help with keeping stool charts, monitoring patients, and collecting stool samples; Manfred Almeida and the staff in the division of microbiology, Hammersmith Hospitals Trust, for help with lactobacilli counts and $C$ difficile toxin tests; and Ruth Peters for organising data entry.

Contributors: ALD'S, MH, CJB, CR, SW, and TRR were all involved in the preparation of the protocol; ALD'S, CJB, and MH obtained ethical approval; ALD'S, CJB, CR, and TRR obtained funding; MH, NM, and ALD'S recruited patients and collected data; $\mathrm{MH}$ managed the running of the trial; TRR set up and SW undertook the microbiological investigations; MH and ALD'S undertook data cleaning and analysis with the assistance of CR and CJB; all authors assisted in the production of the manuscript and interpretation of the results and all approved the final version. $\mathrm{MH}$ is guarantor.

Funding: Healthcare Foundation and Hammersmith Hospital Trustees research committee and Danone Vitapole (Paris, France). The Healthcare Foundation made initial comments on the design of the study. Once funding was agreed none of the funding sources had any role in the data collection, analysis, interpretation of data, writing of the report, or the decision to submit the paper for publication.

Competing interests: $\mathrm{CJB}, \mathrm{MH}$, and ALD'S have received funding from Danone to attend Danone International Conventions on Probiotics. CJB is a member of Danone UK advisory group.

Ethical approval: London multicentre research ethics committee.
10 Leplingard A, Oozeer R, Michelin R, Mogenet A, Seksek I, Diop L, et al. Persistence of living Lactobacillus casei in human stools after regular ingestion of fermented milk. 9th European Nutrition Conference. Rome: Federation of European Nutrition Societies (FENS), Italian Nutrition Society (SINU), 2003.

11 Kelly CP, LaMont JT. Clostridium difficile infection. Annu Rev Med 1998;49:375-90.

12 Dunne C, O'Mahony L, Murphy L, Thornton G, Morrissey D, 0 'Halloran $S$, et al. In vitro selection criteria for probiotic bacteria of human origin: correlation with in vivo findings. Am J Clin Nutr 2001;73:386-92S.

13 Black FT, Anderson PL, Orskov J, Gaarslev K, Lauland S. Prophylactic efficacy of Lactobacilli on traveller's diarrhoea. Travel Medicine 1989;7:333-5.

14 Gotz V, Romankiewicz JA, Moss J, Murray HW. Prophylaxis against ampicillin-associated diarrhea with a lactobacillus preparation. Am J Hosp Pharm 1979;36:754-7.

15 Majamaa H, Isolauri E, Saxelin M, Vesikari T. Lactic acid bacteria in the treatment of acute rotavirus gastroenteritis. J Pediatr Gastroenterol Nutr 1995;20:333-8.

16 Pedone CA, Bernabeu AO, Postaire ER, Bouley CF, Reinert P. The effect of supplementation with milk fermented by Lactobacillus casei (strain DN-114 001) on acute diarrhoea in children attending day care centres. Int J Clin Pract 1999;53:179-84.

17 Pedone CA, Arnaud CC, Postaire ER, Bouley CF, Reinert P. Multicentric study of the effect of milk fermented by Lactobacillus casei on the incidence of diarrhoea. Int J Clin Pract 2000;54:568-71.

18 Saavedra JM, Bauman NA, Oung I, Perman JA, Yolken RH. Feeding of Bifidobacterium bifidum and Streptococcus thermophilus to infants in hospital for prevention of diarrhoea and shedding of rotavirus. Lancet 1994:344:1046-9.

19 Tankanow RM, Ross MB, Ertel IJ, Dickinson DG, McCormick LS, Garfinkel JF. A double-blind, placebo-controlled study of the efficacy of Lactinex in the prophylaxis of amoxicillin-induced diarrhea. DICP 1990;24:382-4.

20 Guarner F, Schaafsma GJ. Probiotics. Int J Food Microbio 1998;39:237-8.

21 Kyne L, Hamel MB, Polavaram R, Kelly CP. Health care costs and difficile. Clin Infect Dis 2002;34:346-53.

22 Wilcox MH, Cunniffe JG, Trundle C, Redpath C. Financial burden of hospital-acquired Clostridium difficile infection. J Hosp Infect 1996;34:23-30.

Accepted: 11 May 2007 mortality associated with nosocomial diarrhea due to Clostridium 